\title{
Clinical significance of rituximab infusion-related reaction in diffuse large B-cell lymphoma patients receiving $\mathrm{R}-\mathrm{CHOP}$
}

\author{
Kyoung Min Cho ${ }^{1,2}$, Bhumsuk Keam ${ }^{1,3}$, Hyerim $\mathrm{Ha}^{1}$, Miso Kim ${ }^{1}$, Jae-Woo Jung ${ }^{4}$, Woo-Jung Song ${ }^{1}$, \\ Tae Min Kim ${ }^{1,3}$, Yoon Kyung Jeon ${ }^{5}$, Hye-Ryun Kang ${ }^{1}$, Dong-Wan Kim ${ }^{1,3}$, Chul Woo Kim5, and Dae Seog Heo ${ }^{1,3}$
}

\begin{abstract}
${ }^{1}$ Department of Internal Medicine, Seoul National University Hospital, Seoul; ${ }^{2}$ Department of Internal Medicine, Kyung Hee University Graduate School of Medicine, Seoul; ${ }^{3}$ Cancer Research Institute, Seoul National University College of Medicine, Seoul; ${ }^{4}$ Department of Internal Medicine, Chung-Ang University College of Medicine, Seoul; ${ }^{5}$ Department of Pathology, Seoul National University Hospital, Seoul, Korea
\end{abstract}

Received: January 20, 2017 Revised : September 12, 2017 Accepted: October 27, 2017

\author{
Correspondence to \\ Bhumsuk Keam, M.D. \\ Department of Internal Medicine, \\ Seoul National University Hos- \\ pital, 101 Daehak-ro, Jongno-gu, \\ Seoul 03080, Korea \\ Tel: $+82-2-2072-7215$ \\ Fax +82-2-762-9662 \\ E-mail:bhumsuk@snu.ac.kr
}

Background/Aims: This study was to evaluate the clinical significance of infusion-related reaction (IRR) of rituximab in diffuse large B-cell lymphoma (DLBCL) patients who received R-CHOP (rituximab, cyclophosphamide, doxorubicin, vincristine, and prednisolone) as a first-line chemotherapy.

Methods: The medical records of 326 patients diagnosed with DLBCL were re trospectively analyzed. Both doctor's progress records and nursing records were reviewed. IRR was graded according to the National Cancer Institute Common Terminology Criteria.

Results: IRR was not associated with overall survival (OS) or progression-free survival (PFS) of DLBCL patients as compared to those who did not have IRR (OS: median 78.0 months vs. 69.0 months, $p=0.700$; PFS: median 65.4 months vs. 64.0 months, $p=0.901$ ). IRR grade did not affect OS or PFS. B symptoms was independently associated with IRR (hazard ratio [HR], 1.850; 95\% confidence interval [CI], 1.041 to 3.290; $p=0.036)$. Further, bone marrow involvement was independently associated with re-IRR (HR, 4.904; 95\% CI, 0.767 to $3.118 ; p=0.029$ ).

Conclusions: Our study shows that IRR of rituximab is not associated with OS or PFS of DLBCL patients who received R-CHOP. Furthermore, our study suggests a need for more careful observation for IRR in patients with B symptoms or bone marrow involvement.

Keywords: Infusion-related reaction; Rituximab; Lymphoma, large B-cell, diffuse; Prognosis; Characteristics

\section{INTRODUCTION}

Diffuse large B-cell lymphoma (DLBCL) is the most common histologic subtype of non-Hodgkin lymphoma (NHL), accounting for approximately $30 \%$ of patients with NHL [1-3]. R-CHOP (comprising rituximab, cyclophosphamide, doxorubicin, vincristine, and prednisolone) is the standard treatment for DLBCL [4-8]. Rituximab targets the $\mathrm{CD} 20$ protein, which is primarily found on the surface of B-cells and is present on many lymphoma cells [9].

Antibody-dependent cellular cytotoxicity (ADCC) is the main mechanism of action of rituximab. ADCC is thought to induce lysis of lymphoma cells via release of cytotoxic mediators in natural killer (NK) cells and macrophages or by direct phagocytosis in macrophages [10]. Further, rituximab has been shown to activate NK cells, macrophages, and neutrophils that eliminate B-cells in 
vitro [11-13]. The T-cell, macrophage, and stromal microenvironments in B-NHL have been extensively investigated, with many studies indicating that patients with enhanced host immune responses may have better clinical outcomes [14].

The infusion-related reaction (IRR) induced by rituximab is one of the most frequently encountered adverse reactions in clinical practice [15]. Most reactions to chemotherapeutic agents are consistent with type 1 hypersensitivity caused by the immunoglobulin E-mediated release of histamines, also which is known decrease of cancer risk by expelling carcinogens. However, IRR induced by rituximab is related to cytokine release $[16,17]$. Recently, small numbered studies suggested that IRR of rituximab is related to its therapeutic effect, with better tumor destruction seen in patients who experienced IRR $[16,18]$. Based on previous reports, we hypothesized that IRR of rituximab may be associated with ADCC, which improves the therapeutic efficacy of rituximab. As previous reports were based on small numbers of patients, the clinical significance of IRR of rituximab in DLBCL patients is not yet fully evaluated. Thus, we investigated the clinical significance of IRR of rituximab in DLBCL patients who received R-CHOP as a first-line chemotherapy.

\section{METHODS}

\section{Patients}

We analyzed DLBCL patients who were diagnosed at Seoul National University Hospital (SNUH) between January 2005 and May 2012 from our consecutive database. The patients who had met the following criteria were enrolled: (1) histologically confirmed DLBCL according to World Health Organization criteria [19] (histologic examination was done by specialized hematopathologists (Y.K. Jeon and C.W. Kim); (2) received R-CHOP as first-line chemotherapy; (3) staging work-up included computed tomography (CT) scans, 18F-2-fluoro-2-deoxyglucose-positron emission tomography/CT, and bone marrow (BM) examination; and (4) We exclude the patients with initial central nervous system involvement, human immunodeficiency virus-associated DLB$\mathrm{CL}$, and intravascular large B-cell lymphoma histology.

The medical record of each patient was reviewed with respect to age at diagnosis, sex, bulky lesion (defined as a longest diameter of nodal mass is more than 10 $\mathrm{cm}$ in size or a widening of the mediastinum by more than one-thirds), Eastern Cooperative Oncology Group (ECOG) performance status, lactate dehydrogenase (LDH) levels, Ann Arbor stage, number of extra-nodal sites, and International Prognostic Index [20].

\section{Rituximab infusion}

All patients received $375 \mathrm{mg} / \mathrm{m}^{2}$ rituximab as an intravenous infusion on day 1 of each chemotherapy cycle, followed by combination chemotherapy. Thirty minutes before rituximab infusion, patients were given $650 \mathrm{mg}$ acetaminophen and $4 \mathrm{mg}$ of the antihistamine pheniramine via intravenous infusion. We did not use steroid premedication due to medication prednisolone.

Rituximab mixed with $500 \mathrm{~mL}$ normal saline was administered at an initial rate of $50 \mathrm{mg} / \mathrm{hr}$, and the rate was increased by $50 \mathrm{mg} / \mathrm{hr}$ every 30 minutes until it reached $400 \mathrm{mg} / \mathrm{hr}$. If no IRR occurred during the first cycle, rituximab infusion during the second and all subsequent cycles started at a rate of $100 \mathrm{mg} / \mathrm{hr}$ followed by increases of $100 \mathrm{mg} / \mathrm{hr}$ every 30 minutes up to $400 \mathrm{mg} / \mathrm{hr}$ for up to 6 to 8 cycles.

Table 1. Definition of infusion-related reaction grade

\begin{tabular}{ll}
\hline Grade & \multicolumn{1}{c}{ Definition } \\
\hline Grade 1 & Mild transient reaction; infusion interruption not indicated; intervention not indicated \\
Grade 2 & $\begin{array}{c}\text { Therapy or infusion interruption indicated but responds promptly to symptomatic treatment (i.e., antihis- } \\
\text { tamines, NSAIDs, narcotics, intravenous fluids); prophylactic medication indicated for less than or equal to } \\
\end{array}$ \\
& $\begin{array}{c}\text { Prolonged (i.e., not rapidly responsive to symptomatic medication and/or brief interruption of infusion); re- } \\
\text { currence of symptoms following initial improvement; hospitalization indicated for other clinical sequelae }\end{array}$ \\
Grade 4 & Life-threatening consequences; urgent intervention indicated \\
Grade 5 & Death
\end{tabular}

NSAID, nonsteroidal anti-inflammatory drug. 


\section{Medical records and grading of infusion-related reactions}

The IRR during first rituximab infusion was included. For each rituximab infusion, both doctor's progress records and nursing records were reviewed. Any change of infusion rate, development of IRR, characteristics of IRR, the exact time and infusion rate of rituximab, and vital signs were recorded in detail. Furthermore, in our hospital there is system which monitoring of adverse drug reaction, which we used. IRR was graded according to the National Cancer Institute Common Terminology Criteria for Adverse Events version 4.o. Definitions of each grade are shown in Table 1. We reviewed IRR relapse rates, the total number of IRR episodes in each patient, and symptoms.

\section{Statistical analysis}

Statistical analyses of categorical variables were performed using Pearson's chi-square tests. Progression-free survival (PFS) was defined as the interval between the date of R-CHOP to the date of disease progression, any cause of death, or the last follow-up visit. Overall survival (OS) was measured from the initiation date of R-CHOP to the date of any cause of death or the last follow-up visit. The median duration of PFS and OS was calculated using the Kaplan-Meier method. Survival comparisons between groups were performed using log-rank tests. The Cox proportional hazards regression model was used for multivariate analyses to assess the effect of patient characteristics and other prognostic factors. Two-sided $p$ values less than 0.05 were considered statistically significant. All analyses were performed using SPSS software for Windows version 19 (IBM Co., Armonk, NY, USA). The Institutional Review Board (IRB) of SNUH approved the study protocol (IRB approval number H-1308-030-510). We also adhered to the Declaration of Helsinki regarding biomedical research involving human subjects. Informed consents were waived because of retrospective study.

\section{RESULTS}

\section{Patient characteristics}

Patient characteristics are summarized in Table 2. A total of 326 DLBCL patients who received first-line R-CHOP
Table 2. Baseline patient characteristics

\begin{tabular}{|c|c|}
\hline Baseline characteristic & Value \\
\hline Age, yr & $63(17-94)$ \\
\hline \multicolumn{2}{|l|}{ Sex } \\
\hline Female & $169(51.8)$ \\
\hline Male & $157(48.2)$ \\
\hline \multicolumn{2}{|c|}{ Eastern Cooperative Oncology Group } \\
\hline $0-1$ & $268(82.2)$ \\
\hline $2-4$ & $39(12.0)$ \\
\hline Unknown & $19(5.8)$ \\
\hline \multicolumn{2}{|l|}{ Ann Arbor stage } \\
\hline I & $50(15 \cdot 3)$ \\
\hline II & $118(36.2)$ \\
\hline III & $56(17.2)$ \\
\hline IV & $102(31.3)$ \\
\hline \multicolumn{2}{|l|}{ B symptom } \\
\hline Present & $251(77.0)$ \\
\hline Absent & $75(23.0)$ \\
\hline \multicolumn{2}{|l|}{ No. of extranodal site } \\
\hline$<2$ & $146(44.8)$ \\
\hline$>2$ & $180(55.2)$ \\
\hline \multicolumn{2}{|l|}{ Lactate dehydrogenase } \\
\hline Normal & $121(37.1)$ \\
\hline Elevated & $203(62.3)$ \\
\hline Unknown & $2(0.6)$ \\
\hline \multicolumn{2}{|l|}{ Bone marrow } \\
\hline Not involved & $262(80.4)$ \\
\hline Involved & $54(16.6)$ \\
\hline Unknown & $10(3.0)$ \\
\hline \multicolumn{2}{|l|}{ Bulky lesion } \\
\hline Present & $74(22.7)$ \\
\hline Absent & $250(76.7)$ \\
\hline Not evaluable & $2(0.6)$ \\
\hline \multicolumn{2}{|c|}{ International Prognostic Index } \\
\hline 0 & $52(16.0)$ \\
\hline 1 & $96(29.4)$ \\
\hline 2 & $82(25 \cdot 2)$ \\
\hline 3 & $57(17 \cdot 5)$ \\
\hline 4 & $30(9.2)$ \\
\hline 5 & $9(2.8)$ \\
\hline \multicolumn{2}{|l|}{ Best response } \\
\hline Complete remission & $235(72.1)$ \\
\hline Partial response & $51(15.6)$ \\
\hline Stable disease & $8(2.5)$ \\
\hline Progression disease & $7(2.1)$ \\
\hline Not evaluable & $25(7.7)$ \\
\hline
\end{tabular}

Values are presented as median (range) or number (\%). 
Table 3. Infusion-related reaction during rituximab infusion (per-person)

\begin{tabular}{|c|c|}
\hline Variable & No. (\%) \\
\hline \multicolumn{2}{|l|}{ IRR } \\
\hline Absent & $174(56.7)$ \\
\hline Present & $133(43 \cdot 3)$ \\
\hline \multicolumn{2}{|l|}{ Acute IRR grade } \\
\hline Grade o-1 & $197(64.2)$ \\
\hline Grade 2 & $97(31.6)$ \\
\hline Grade $3-4$ & $13(4.2)$ \\
\hline \multicolumn{2}{|l|}{ IRR re-experiences after 1st IRR } \\
\hline Absent & $288(93.8)$ \\
\hline Present & $19(6.2)$ \\
\hline \multicolumn{2}{|l|}{ Total no. of IRR in same patient } \\
\hline 0 & $187(60.9)$ \\
\hline 1 & $97(31.6)$ \\
\hline 2 & $17(5 \cdot 5)$ \\
\hline 3 & $5(1.6)$ \\
\hline 4 & 0 \\
\hline 5 & o \\
\hline 6 & $1(0.3)$ \\
\hline \multicolumn{2}{|l|}{ Symptom of hypersensitivity } \\
\hline Only itching & $30(9.8)$ \\
\hline Respiratory symptom & $8(2.6)$ \\
\hline Myalgia & $4(1.3)$ \\
\hline Urticaria & $23(7 \cdot 5)$ \\
\hline Hypoxia & $1(0.3)$ \\
\hline Tingling & $1(0.3)$ \\
\hline Rash & $6(2.0)$ \\
\hline Angioedema & $6(2.0)$ \\
\hline Nasal obstruction & $7(2.3)$ \\
\hline Cough & $1(0.3)$ \\
\hline Fever & $17(5 \cdot 5)$ \\
\hline Chilling & $56(18.2)$ \\
\hline Chest discomfort & $4(1.3)$ \\
\hline Dyspnea & $8(2.6)$ \\
\hline Dizziness & $12(3 \cdot 9)$ \\
\hline Flushing & $10(3 \cdot 3)$ \\
\hline Arrhythmia & o \\
\hline Headache & $14(4.6)$ \\
\hline Subconjunctival hemorrhage & $2(0.7)$ \\
\hline Hypotension & $11(3.6)$ \\
\hline Neurologic symptom & $29(9.4)$ \\
\hline Nausea/vomiting & $15(4 \cdot 9)$ \\
\hline ICU care & o \\
\hline Death & o \\
\hline
\end{tabular}

IRR, Infusion-related reaction; ICU, intensive care unit. were analyzed. The median age was 63 years (range, 17 to 94). One hundred and fifty-eight patients (48.5\%) were in advanced stages of disease (stages III and IV). Two hundred and three patients (62.3\%) had elevated LDH levels at the time of diagnosis. Seventy-four patients (22.7\%) had bulky lesions.

\section{Infusion-related reactions}

One hundred and thirty-three patients (43.3\%) experienced one or more than IRR event(s) (Table 3). Twelve patients had Grade 3 IRR and one patient had Grade 4 IRR. Fifty-six patients (18.2\%) experienced chills, 15 patients (4.9\%) experienced nausea and vomiting, and 12 patients $(3.9 \%)$ experienced hypoxia and hypotension. No patient required intensive care unit admission or died from IRR. Nineteen patients (6.2\%) experienced two IRR re-experiences. Twenty-three (7.4\%) experienced more than two IRR re-experiences.

\section{Risk factors for IRR and IRR re-experience}

Among the clinical parameters investigated, B symptoms were significantly associated with the incidence of IRR (hazard ratio [HR], 1.850; 95\% confidence interval [CI], 1.041 to 3.290; $p=0.036$ ). However, lymphoma stage, the number of extra-nodal lesions and LDH had no relation with the incidence of IRR. Furthermore, response rate of R-CHOP was not different in IRR and non-IRR groups (HR, 1.697; 95\% CI, 0.549 to 5.243; $p=0.284$ ) (Table 4). BM involvement was significantly associated with the incidence of IRR re-experience (HR, 4.904; 95\% CI, 0.767 to $3.118 ; p=0.029)$. B symptoms, lymphoma stage, extra-nodal number, and treatment response had no relation to the incidence of IRR re-experiences (Table 5).

\section{Clinical outcome and correlation with IRR}

OS was not significantly longer in patients who experienced IRR compared to those who did not experience IRR (median, 67.5 months vs. 78.0 months, $p=0.975$ ) (Fig. 1). PFS was also not significantly different between these two groups (median, 65.4 months vs. 63.0 months, $p=0.770$ ) (Fig. 2). Also, IRR is not associated with the therapeutic response rate $(p=0.361)$. OS was not significantly different in patients with IRR grades of o or 1, and those with IRR grades of 2 and 3, or 4 (median, 79.2 months vs. 65.7 months vs. 61.9 months, $p=0.848$ ) (Fig. 3). PFS was also not significantly different between these three groups 
Table 4. Baseline characteristics of IRR and non-IRR patients

\begin{tabular}{lllc}
\hline Characteristic & HR & 95\% CI & $p$ value \\
\hline Stage (III, IV) & 0.774 & $0.435-1.376$ & 0.383 \\
No. of extranodal site (> 2) & 1.292 & $0.777-2.150$ & 0.325 \\
B symptom (present) & 1.850 & $1.041-3.290$ & 0.036 \\
LDH (elevated) & 0.931 & $0.554-1.565$ & 0.787 \\
Bulky lesion (present) & 0.893 & $0.494-1.614$ & 0.707 \\
BM (involved) & 1.546 & $0.767-3.118$ & 0.478 \\
ORR (SD or PD) & 1.697 & $0.549-5.243$ & 0.284 \\
\hline
\end{tabular}

IRR, infusion-related reaction; HR, hazard ratio; CI, confidence interval; LDH, lactate dehydrogenase; BM, bone marrow; ORR, objective response rate; $\mathrm{SD}$, stable disease; $\mathrm{PD}$, progression disease.

Table 5. Baseline characteristics of IRR and IRR re-experience patients

\begin{tabular}{lccc}
\hline Characteristic & HR & 95\% CI & $p$ value \\
\hline Stage (III, IV) & 0.929 & $0.215-4.019$ & 0.922 \\
No. of extranodal site (> 2) & 0.888 & $0.251-4.019$ & 0.854 \\
B symptom (present) & 0.667 & $0.193-2.309$ & 0.523 \\
LDH (elevated) & 1.386 & $0.428-4.483$ & 0.586 \\
Bulky lesion (present) & 1.736 & $0.513-5.871$ & 0.375 \\
BM (involved) & 4.904 & $0.767-3.118$ & 0.029 \\
ORR (SD or PD) & 0.897 & $0.093-8.617$ & 0.996 \\
\hline
\end{tabular}

IRR, infusion-related reaction; HR, hazard ratio; CI, confidence interval; LDH, lactate dehydrogenase; BM, bone marrow; ORR, objective response rate; $\mathrm{SD}$, stable disease; $\mathrm{PD}$, progression disease.

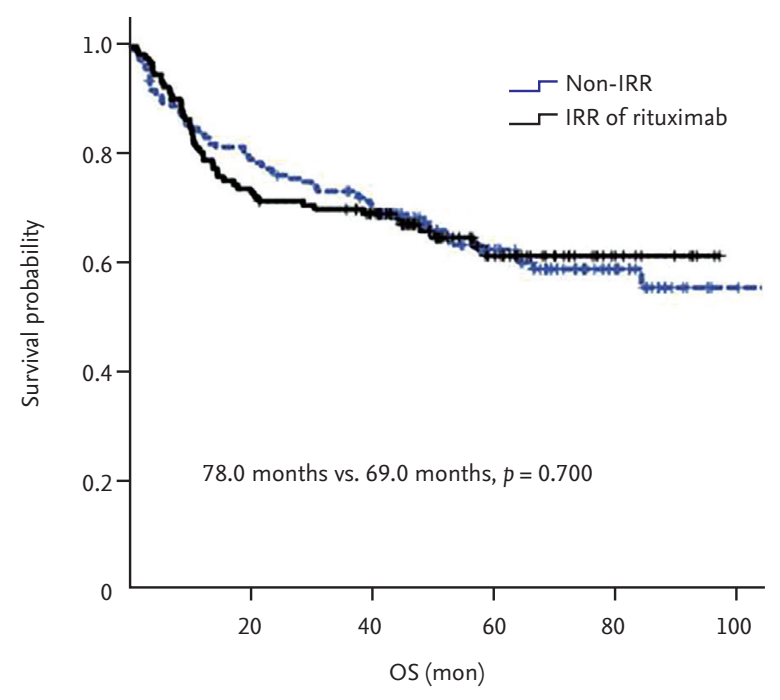

Figure 1. Kaplan-Meier plots of overall survival (OS) by infusion-related reaction (IRR) and non-IRR.

(median, 65.6 months vs. 63.7 months vs. 48.0 months, $p=$ 0.388) (Fig. 3). OS was not significantly different between patients who re-experienced IRR and those who did not

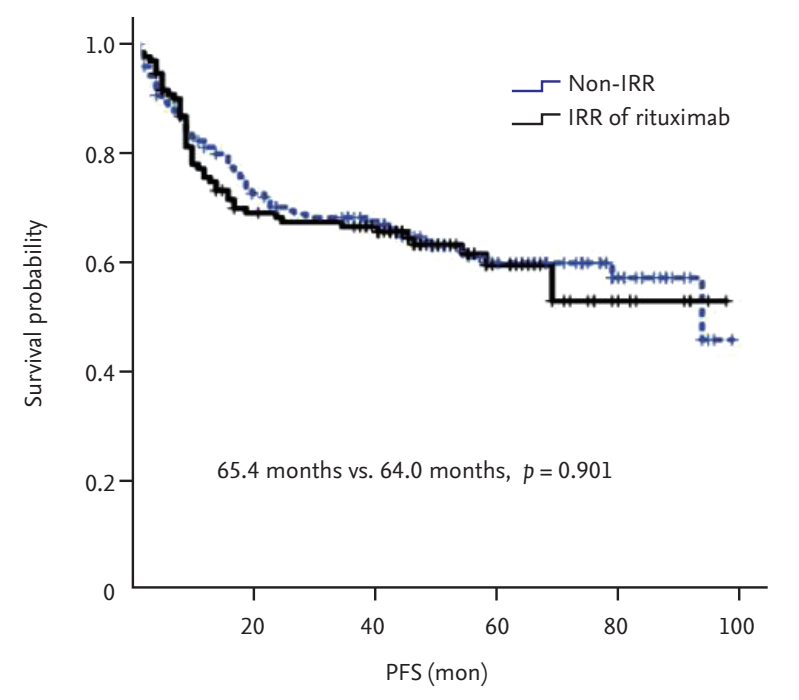

Figure 2. Kaplan-Meier plots of progression-free survival (PFS) by infusion-related reaction (IRR) and non-IRR.

(median, 78.9 months vs. 48.4 months, $p=0.255$ ). However, PFS was significantly different between these two groups (median, 65.8 months vs. 35.4 months, $p=0.046$ ). 

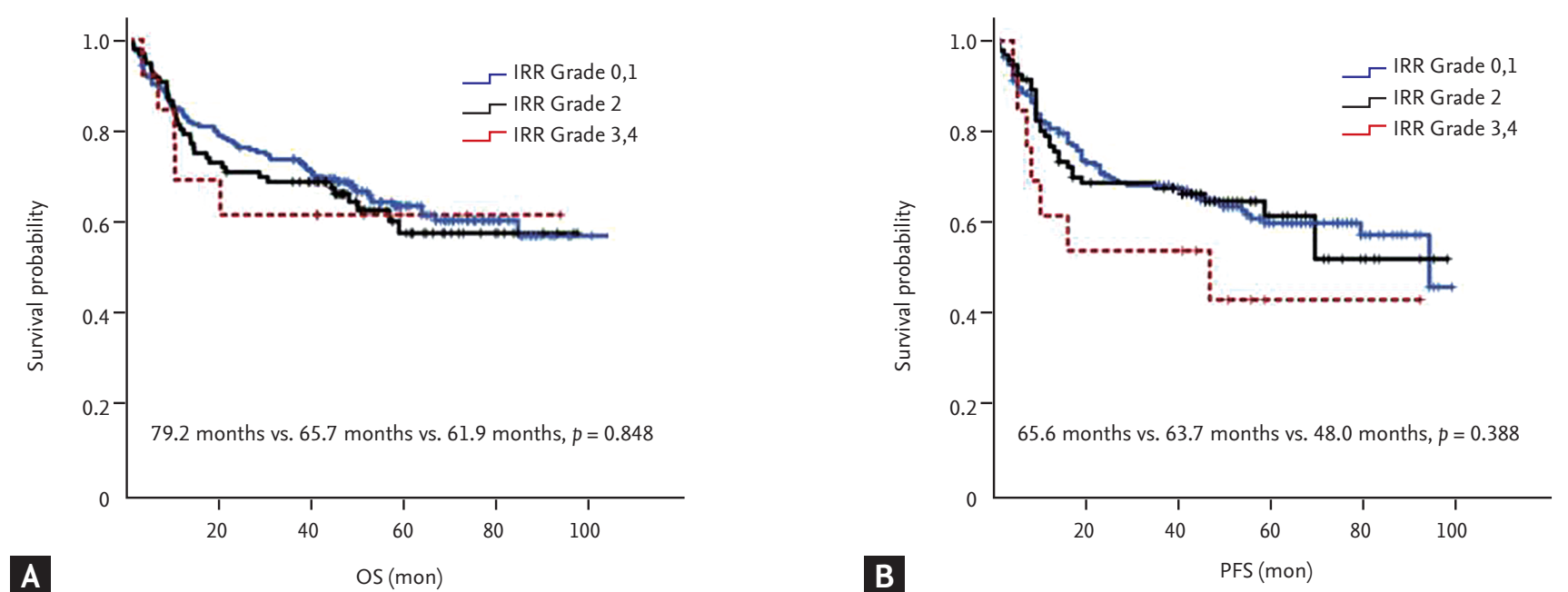

Figure 3. Kaplan-Meier plots of overall survival (OS) and progression-free survival (PFS) by infusion-related reaction (IRR) grades.

In IRR group analysis, there was not different between $\mathrm{BM}$ involvement and not BM involvement (median OS, 68.2 months vs. 61.5 months, $p=0.491)$. However, there was different between B symptom and not B symptom (median OS, 72.8 months vs. 53.3 months, $p=0.019$ ).

\section{DISCUSSION}

Our study showed that there was no association between IRR of rituximab and OS or PFS. Furthermore, there was no association between IRR of rituximab and response rate to R-CHOP. B symptoms was independently associated with IRR of rituximab. Therefore, patients with B symptoms should be more intensively monitored for IRR of rituximab.

Mild-to-moderate reactions are relatively common (up to $77 \%$ ) particularly during the first infusion of rituximab. The reactions are immediate, often occurring within the first few minutes of the first infusion [17]. As our results show that IRR experience is related to B symptoms, during a challenge it may help to decide which patients might benefit from a reduction in the infusion rate of rituximab by half(from 100 to $50 \mathrm{mg} / \mathrm{hr}$ ) [17].

Rituximab is thought to induce lysis of lymphoma cells through complement-mediated cytolysis, ADCC, and direct induction of apoptosis. Several previous reports suggest a link between IRR of rituximab and its therapeutic efficacy. Byrd et al. [16] reported that all pa- tients who developed a unique set of severe IRRs subsequently showed a rapid decrement in circulating tumor cell load. Also, Winkler et al. [15] reported that serum levels of interleukin 6 and tumor necrosis factor $\alpha$ peaked after the onset of infusion, peak levels were accompanied by maximum clinical side effects, and lymphocyte counts dropped to $50 \%$ to $75 \%$ of baseline values within 12 hours after infusion onset. Unlike above reports from small numbers of patients, our results from a larger number of DLBCL patients showed no association between IRR of rituximab and the therapeutic efficacy of rituximab. Recent reports suggest that IRR is not associated with the therapeutic efficacy of rituximab [21]. The IRR of rituximab was related only to B symptoms in our study. It is otherwise that previous report result which reported that BM involvement is associated with IRR [21].

Further, B symptoms are known for poor prognosis [22-24]. Also, our study showed that patients with B symptom had significantly poor OS in univariate analysis $(p<0.001)$. However, our study also showed that the patients with IRR of rituximab did not have poorer OS than the patients without IRR of rituximab. Considering the above, B symptom itself are not related to prognosis of IRR. Hong et al. [21] reported that patients with DLBCL who experienced $\geq$ Grade 2 IRR had reduced eventfree and OS rates compared to those who did not experience IRR of rituximab. However, our study showed that IRR grade is not associated with therapeutic prognosis. 
Furthermore, in the study of Hong et al. [21], they reported approximately $18 \%$ of IRR of rituximab in DLBCL. However, our study showed an unexpectedly high rate of IRR ( $43 \%)$. In our study, we did not use premedication steroid because our chemotherapy include prednisolon $100 \mathrm{mg}$, as a result, our study showed an unexpectedly high rate of IRR due to the non-use of premedication steroid.

Our study showed that although, OS was not significantly different between patients who re-experienced IRR and those who did not (78.9 months vs. 48.4 months, $p=0.255)$. However, PFS was significantly different between these two groups $(p=0.046)$. BM involvement was significantly associated with the incidence of IRR re-experience $(p=0.029)$. BM involvement is known for poor prognosis [22-24]. Our study showed that patients with $\mathrm{BM}$ involvement had significantly poor PFS in univariate analysis $(p=0.02)$. Van der Kolk et al. [25] recently reported that complement activation plays a key role in the side effects of rituximab treatment. Additionally, this study also reported that complement activation was only observed in patients with BM involvement [25]. Furthermore, Van der Kolk et al. [25] claimed that patients with BM involvement might have higher levels of circulating tumor cells than patients without BM involvement. Several other reports suggest that the incidence and severity of side effects associated with the first dose of rituximab significantly depend on higher circulating tumor lymphocyte counts at baseline in patients with a variety of other lymphoid neoplasms, such as chronic lymphocytic leukemia and B-cell chronic lymphocytic leukemia $[15,16,26]$. Although we could not evaluate blood lymphocytes in this study as all patients had DLBCL without leukemic manifestations, we observed that the $\mathrm{BM}$ involvement is associated with rituximab-related re-experience IRR. This is consistent with our observation that rituximab-related re-experience IRR by itself affect IRR-related treatment efficiency.

Although, we could not check changes in cytokine during IRR. To the best of our knowledge, our study is the largest study with enough statistical power for evaluating the clinical significance of IRR of rituximab in DLBCL patients receiving R-CHOP. Previous reports studied smaller population sizes $(n=17$ and $n=5)$ with other B malignancies such as B-cell prolymphocytic leukemia and transformed NHL [15,16]. Moreover, all the patients in our study received rituximab infusion in the same protocol in a single center. However, there are several limitations to our study. It is important to note that all the data in our study were collected retrospectively. We reviewed IRR according to doctor's progress records and nursing records, so there are possibility of underestimation of lower grade IRR, variation according to the investigators. Also, the levels of cytokines, neutrophils, or macrophages were not evaluated during IRR of rituximab.

In conclusion, the incidence of B symptoms were independently associated with IRR of rituximab. Our study shows that IRR of rituximab is not associated with OS or PFS in DLBCL patients who received R-CHOP. Further, BM involvement is associated with re-IRR of rituximab. Therefore, patients with B symptoms and $\mathrm{BM}$ involvement should be more intensively monitored for IRR of rituximab.

\section{KEY MESSAGE}

1. Infusion-related reaction (IRR) of rituximab is not associated with overall survival or progression-free survival of diffuse large B-cell lymphoma patients who received R-CHOP (comprising rituximab, cyclophosphamide, doxorubicin, vincristine, and prednisolone).

2. Our study suggests a need for more careful monitoring for IRRs in patients with B symptoms or bone marrow involvement.

\section{Conflict of interest}

No potential conflict of interest relevant to this article was reported.

\section{Acknowledgments}

This study was supported by grants from the Innovative Research Institute for Cell Therapy, Republic of Korea (Ao62260).

\section{REFERENCES}

1. Harris NL, Jaffe ES, Stein H, et al. A revised European-American classification of lymphoid neoplasms: 
a proposal from the International Lymphoma Study Group. Blood 1994;84:1361-1392.

2. Harris NL, Jaffe ES, Diebold J, et al. World Health Organization classification of neoplastic diseases of the hematopoietic and lymphoid tissues: report of the Clinical Advisory Committee meeting-Airlie House, Virginia, November 1997. J Clin Oncol 1999;17:3835-3849.

3. Armitage JO, Weisenburger DD. New approach to classifying non-Hodgkin's lymphomas: clinical features of the major histologic subtypes. Non-Hodgkin's Lymphoma Classification Project. J Clin Oncol 1998;16:2780-2795.

4. Coiffier B, Lepage E, Briere J, et al. CHOP chemotherapy plus rituximab compared with CHOP alone in elderly patients with diffuse large-B-cell lymphoma. N Engl J Med 2002;346:235-242.

5. Habermann TM, Weller EA, Morrison VA, et al. Rituximab-CHOP versus CHOP alone or with maintenance rituximab in older patients with diffuse large B-cell lymphoma. J Clin Oncol 2006;24:3121-3127.

6. Pfreundschuh M, Schubert J, Ziepert M, et al. Six versus eight cycles of bi-weekly CHOP-14 with or without rituximab in elderly patients with aggressive $\mathrm{CD} 2 \mathrm{O}+\mathrm{B}$-cell lymphomas: a randomised controlled trial (RICOVER-60). Lancet Oncol 2008;9:105-116.

7. Pfreundschuh M, Trumper L, Osterborg A, et al. CHOPlike chemotherapy plus rituximab versus CHOP-like chemotherapy alone in young patients with good-prognosis diffuse large-B-cell lymphoma: a randomised controlled trial by the MabThera International Trial (MInT) Group. Lancet Oncol 2006;7:379-391.

8. Sehn LH, Donaldson J, Chhanabhai M, et al. Introduction of combined CHOP plus rituximab therapy dramatically improved outcome of diffuse large B-cell lymphoma in British Columbia. J Clin Oncol 2005;23:5027-5033.

9. McLaughlin P, Grillo-Lopez AJ, Link BK, et al. Rituximab chimeric anti-CD20 monoclonal antibody therapy for relapsed indolent lymphoma: half of patients respond to a four-dose treatment program. J Clin Oncol 1998;16:28252833 .

10. Alduaij W, Illidge TM. The future of anti-CD2o monoclonal antibodies: are we making progress? Blood 2011; 117:2993-3001.

11. Beers SA, Chan CH, James S, et al. Type II (tositumom$\mathrm{ab}$ ) anti-CD2o monoclonal antibody out performs type I (rituximab-like) reagents in B-cell depletion regardless of complement activation. Blood 2008;112:4170-4177.
12. Cartron G, Dacheux L, Salles G, et al. Therapeutic activity of humanized anti-CD2o monoclonal antibody and polymorphism in IgG Fc receptor FcgammaRIIIa gene. Blood 2002;99:754-758.

13. Shibata-Koyama M, Iida S, Misaka H, et al. Nonfucosylated rituximab potentiates human neutrophil phagocytosis through its high binding for FcgammaRIIIb and MHC class II expression on the phagocytotic neutrophils. Exp Hematol 2009;37:309-321.

14. Burger JA, Ghia P, Rosenwald A, Caligaris-Cappio F. The microenvironment in mature B-cell malignancies: a target for new treatment strategies. Blood 2009;114:3367-3375.

15. Winkler U, Jensen M, Manzke O, Schulz H, Diehl V, Engert A. Cytokine-release syndrome in patients with B-cell chronic lymphocytic leukemia and high lymphocyte counts after treatment with an anti-CD2o monoclonal antibody (rituximab, IDEC-C2B8). Blood 1999;94:22172224.

16. Byrd JC, Waselenko JK, Maneatis TJ, et al. Rituximab therapy in hematologic malignancy patients with circulating blood tumor cells: association with increased infusion-related side effects and rapid blood tumor clearance. J Clin Oncol 1999;17:791-795.

17. Lenz HJ. Management and preparedness for infusion and hypersensitivity reactions. Oncologist 2007;12:601-609.

18. Cheson BD, Frame JN, Vena D, Quashu N, Sorensen JM. Tumor lysis syndrome: an uncommon complication of fludarabine therapy of chronic lymphocytic leukemia. J Clin Oncol 1998;16:2313-2320.

19. Campo E, Swerdlow SH, Harris NL, Pileri S, Stein H, Jaffe ES. The 2008 WHO classification of lymphoid neoplasms and beyond: evolving concepts and practical applications. Blood 2011;117:5019-5032.

20. International Non-Hodgkin's Lymphoma Prognostic Factors Project. A predictive model for aggressive non-Hodgkin's lymphoma. N Engl J Med 1993;329:987-994.

21. Hong J, Kim JY, Ahn HK, et al. Bone marrow involvement is predictive of infusion-related reaction during rituximab administration in patients with B cell lymphoma. Support Care Cancer 2013;21:1145-1152.

22. Sharma R, Cunningham D, Smith P, Robertson G, Dent O, Clarke SJ. Inflammatory (B) symptoms are independent predictors of myelosuppression from chemotherapy in Non-Hodgkin Lymphoma (NHL) patients: analysis of data from a British National Lymphoma Investigation phase III trial comparing CHOP to PMitCEBO. BMC 
Cancer 2009;9:153.

23. Legouffe E, Rodriguez C, Picot MC, et al. C-reactive protein serum level is a valuable and simple prognostic marker in non Hodgkin's lymphoma. Leuk Lymphoma 1998;31:351-357.

24. Yan Y, Chan WC, Weisenburger DD, et al. Clinical and prognostic significance of bone marrow involvement in patients with diffuse aggressive B-cell lymphoma. J Clin Oncol 1995;13:1336-1342.
25. van der Kolk LE, Grillo-Lopez AJ, Baars JW, Hack CE, van Oers MH. Complement activation plays a key role in the side-effects of rituximab treatment. Br J Haematol 2001;115:807-811.

26. Lim LC, Koh LP, Tan P. Fatal cytokine release syndrome with chimeric anti-CD2o monoclonal antibody rituximab in a 71-year-old patient with chronic lymphocytic leukemia. J Clin Oncol 1999;17:1962-1963. 